\title{
PROGRAMMABLE OTA-BASED MULTIFUNCTION ACTIVE FILTER
}

\author{
ABDULRAHMAN KHALAF AL-ALI, MUHAMMAD TAHER \\ ABUELMA'ATTI AND RIZWAN ALI TIWANA \\ King Fahd University of Petroleum and Minerals, Box 203 Dhahran 31261 Saudi Arabia \\ (Received December 30, 1993; in final form January 25, 1994)
}

\begin{abstract}
A fully programmable multifunction filter is presented. The filter uses four operational transconductance amplifiers and two capacitors. Analog switches are used to control the filter transfer function through a microcontroller. The user is provided control over the cutoff (or centre) frequency of the filter by controlling the auxiliary bias currents of the operational transconductance amplifiers via digital-toanalog converters. The circuit can simultaneously provide three programmable transfer functions in a single structure and can realize all the standard second-order filters. The circuit is attractive for VLSI implementation.
\end{abstract}

\section{INTRODUCTION}

In a recent publication, $\mathrm{Wu}$ and $\mathrm{Xie}$ [1] proposed a new multifunction active filter using operational transconductance amplifiers (OTAs). The proposed circuit requires only four OTAs and can realize all second-order functions, namely, lowpass (LPF), highpass (HPF), bandpass (BPF), bandeliminate (BEF) and allpass (APF) transfer functions. A very attractive feature of the circuit is that three outputs are simultaneously available from a single structure. Thus, realization of three different filter transfer functions using a single OTA-based circuit is feasible.

On the other hand, the advancement of VLSI technology has increased the density of integration and reduced the size of the circuitry to a large extent. Nowadays, a complete system on a single-chip is highly desirable. Such a system may include both digital and analog circuits.

The major intention of the present paper is, therefore, to investigate the feasibility of using the proposed circuit of $\mathrm{Wu}$ and Xie [1] as a building block for realizing programmable multifunction filter transfer functions. In this regard, the proposed circuit will be integrated with an 8-bit microcontroller and analog switches. The microcontroller is a single chip eight-bit microprocessor that monitors and controls the process. It has built-in RAM, an EPROM, an EEPROM, and an eight-bit ADC. Through the microcontroller, the user is provided complete control over the major filter characteristics via a software menu. 


\section{PROPOSED PROGRAMMABLE FILTER}

Fig. 1 shows the filter configuration proposed by Wu and Xie [1]. Assuming ideal OTAs, it has been shown by Wu and Xie that the proposed filter circuit can provide 42 filter realizations with a single output, 15 filter realizations with two outputs, and three filter realizations with three simultaneous outputs. The major concern of this paper is to investigate the feasibility of obtaining programmable multifunction realization using the circuit of Fig. 1 as a basic building block. Thus, only the three possible realizations that yield three transfer functions simultaneously will be considered in the following investigation. These realizations are summarized in Table I. A " 0 " in the input column of Table I indicates the corresponding input is connected to ground while a " 1 " indicates it is connected to the signal source. For the allpass filter realization obtained with inputs 10110 , the condition of realization is $\mathrm{g}_{1}=\mathrm{g}_{2}$. From Table $\mathrm{I}$, one can see that a particular filter realization can be obtained by properly connecting the input terminals to the signal source or to the ground.

A programmable implementation for connecting input terminals to signal source or to ground is shown in Fig. 2. In Fig. 2, five analog switches are used to control the inputs to the five input terminals of the circuit of Fig. 1. Input to each of the analog switches is the signal source and the ground. Five selection lines of the switches are coupled to an output port of the microcontroller, which is controlled through software. A " 1 " on the selection line results in the corresponding filter input to be connected to the signal source, while a " 0 " on the selection line results in the corresponding input to be grounded. According to user's choice, through a software menu, the microcontroller sends an appropriate digital signal to the output port, thus achieving the proper input connection to the filter section. The switches used ensure that the signal is not digitized and remaines analog. The use of analog switches with the microcontroller allows the user to change the filter inputs at any time through the software, thus giving the user the freedom to select the required output.

Fig. 2 shows also a possible mechanism for controlling the auxiliary bias current $I_{A B C}$ of the OTAs. Since the transconductance of the OTA is a function of the auxiliary bias current, $g=I_{A B C} / 2 V_{T}$, where $V_{T}$ is the thermal voltage, controlling the bias voltage results in a control on the g's of the OTAs and consequently on the parameters $\omega_{0}$ and $\omega_{0} / Q_{0}$ of the filter. Controlling the auxiliary bias currents of the OTAs can be achieved by using a four channel D/A converter connected to the microcontroller. Initially, an equal voltage is established for each OTA. Output of the $\mathrm{D} / \mathrm{A}$ is changed according to user requirement by the software. Each time a request for change is made, the previous value is compared against MAX and MIN operating values prespecified by the user. This check is required for proper operation of the OTAs. In other words, this check will ensure that the maximum and minimum values of the OTA auxiliary bias currents are not exceeded. It must be mentioned, however, that in realizations where the gain of the filter is a function of the OTA's transconductance, a change in the parameters $\omega_{0}$ and/or $\omega_{0} / Q_{0}$ may result in a change in the gain. 


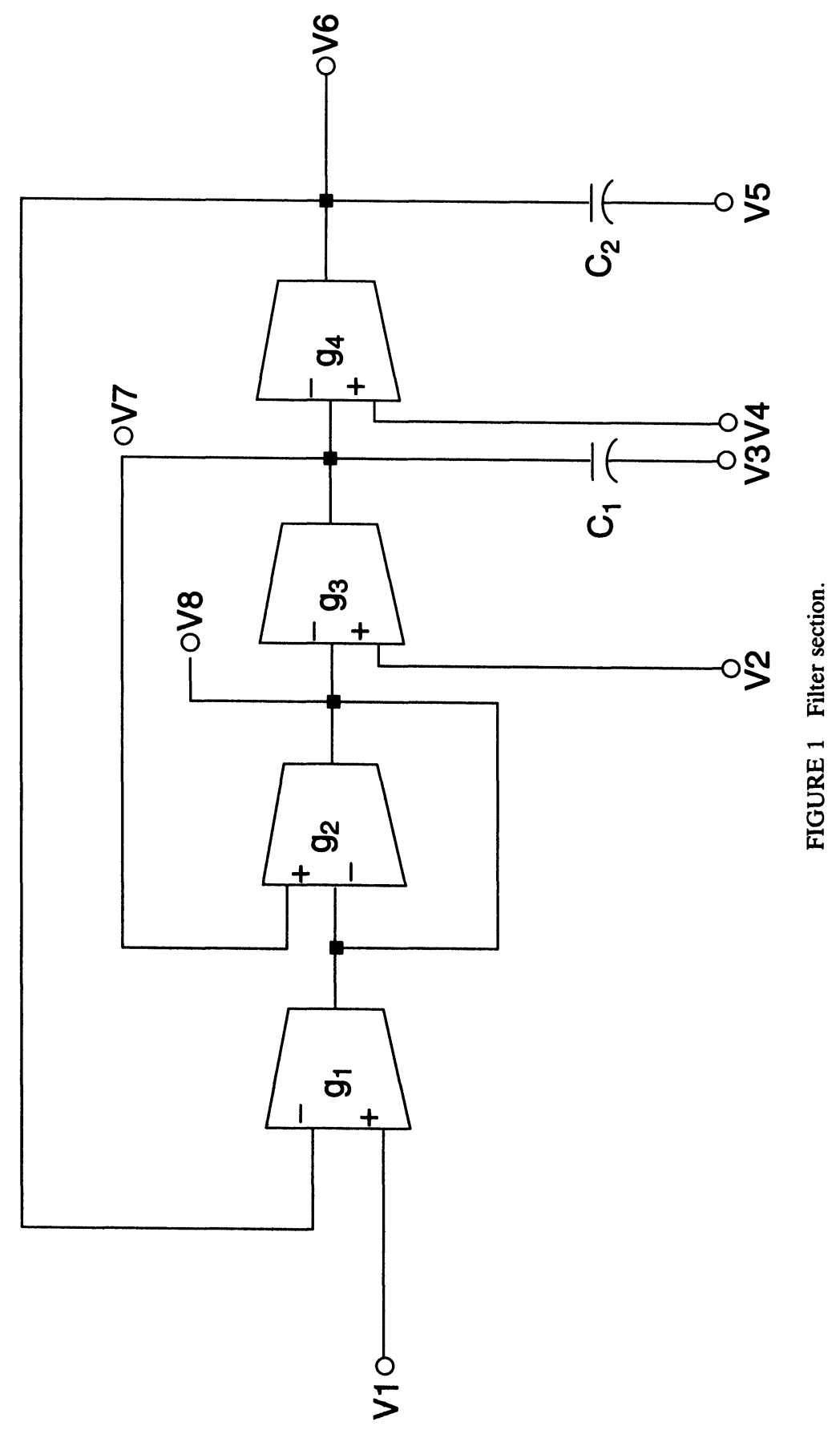


TABLE I

Possible filter realizations.

\begin{tabular}{|c|c|c|c|c|c|c|c|}
\hline \multicolumn{5}{|c|}{ Input Terminals } & \multicolumn{3}{|c|}{ Filter Transfer Function } \\
\hline $\mathrm{V}_{1}$ & $\mathrm{~V}_{2}$ & $\mathrm{~V}_{3}$ & $\mathrm{~V}_{4}$ & $\mathrm{~V}_{5}$ & $\mathrm{~V}_{6}(\mathrm{~s}) / \mathrm{V}_{\mathrm{i}}(\mathrm{s})$ & $\mathrm{V}_{7}(\mathrm{~s}) / \mathrm{V}_{\mathrm{i}}(\mathrm{s})$ & $\mathrm{V}_{8}(\mathrm{~s}) / \mathrm{V}_{\mathrm{i}}(\mathrm{s})$ \\
\hline \multirow[t]{3}{*}{1} & 0 & 0 & 0 & 0 & $\omega_{0}^{2} / \mathrm{D}(\mathrm{s})$ & $-\mathrm{g}_{1} / \mathrm{g}_{2}\left(\frac{\omega_{0}}{\mathrm{Q}_{0}}\right) \mathrm{s}$ & $\frac{\mathrm{g}_{1} / \mathrm{g}_{2} \mathrm{~s}^{2}}{\mathrm{D}(\mathrm{s})}$ \\
\hline & & & & & & $\mathrm{D}(\mathrm{s})$ & \\
\hline & & & & & LPF & $\mathrm{BPF}$ & HPF \\
\hline \multirow[t]{2}{*}{0} & 0 & 1 & 1 & 0 & $\frac{\mathrm{g}_{2} / \mathrm{g}_{1} \omega_{0}^{2}}{\mathrm{D}(\mathrm{s})}$ & $\frac{\mathrm{s}^{2}+\omega_{0}^{2}}{\mathrm{D}(\mathrm{s})}$ & $S^{2} / D(s)$ \\
\hline & & & & & LPF & BEF & HPF \\
\hline \multirow[t]{2}{*}{1} & 0 & 1 & 1 & 0 & $\frac{\left(1+g_{2} / g_{1}\right) \omega_{0}^{2}}{D(s)}$ & $\frac{\mathrm{s}^{2}-\mathrm{s} \frac{\omega_{0}}{\mathrm{Q}_{0}}+\omega_{0}^{2}}{\mathrm{D}(\mathrm{s})}$ & $\frac{\left(1+g_{1} / g_{2}\right) s^{2}}{D(s)}$ \\
\hline & & & & & LPF & APF & HPF \\
\hline
\end{tabular}

$\mathrm{D}(\mathrm{s})=\mathrm{s}^{2}+\frac{\omega_{0}}{\mathrm{Q}_{0}} \mathrm{~s}+\omega_{0}^{2}, \omega_{0}^{2}=\frac{\mathrm{g}_{1} \mathrm{~g}_{3} \mathrm{~g}_{4}}{\mathrm{~g}_{2} \mathrm{C}_{1} \mathrm{C}_{2}}, \mathrm{Q}_{10}=\left(\frac{\mathrm{g}_{1} \mathrm{~g}_{4} \mathrm{C}_{1}}{\mathrm{~g}_{2} \mathrm{~g}_{3} \mathrm{C}_{2}}\right)^{1 / 2}$

\section{SOFTWARE}

The software for the system has been developed in the assembly language of the microcontroller. The flowchart in Fig. 3 outlines the routines involved.

\section{The Initialisation Routine}

a. An input disable signal is sent to the output port, connected to the analog switches, such that, all the filter inputs are grounded.

b. Through $\mathrm{D} / \mathrm{A}$ a voltage equivalent to 5 volts is applied to all the biasing resistors.

c. Look-up table for different realizations is generated.

\section{Menu}

a. Main menu for the program is displayed for the user, through which he can decide the combination of three filter outputs required.

b. After first selection a sub menu is displayed stating the available gains for different realizations.

\section{Analog Switch Control}

a. Once the user has made the selection, a lookup table is scanned through which proper digital output to the analog switches is selected.

b. The chosen value is sent to the output port connected to the selection lines of the analog switches, hence achieving proper input application to the filter section. 


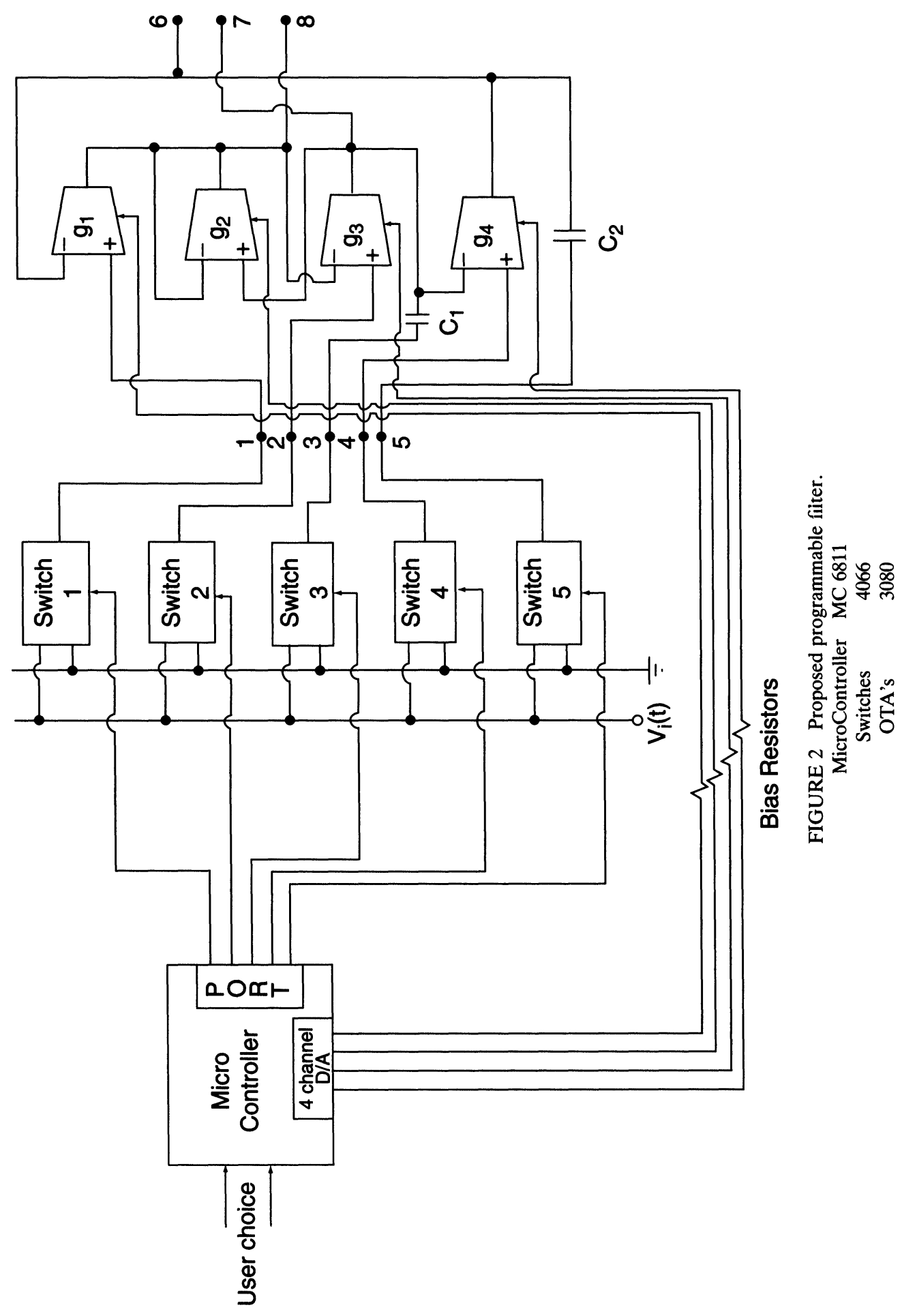




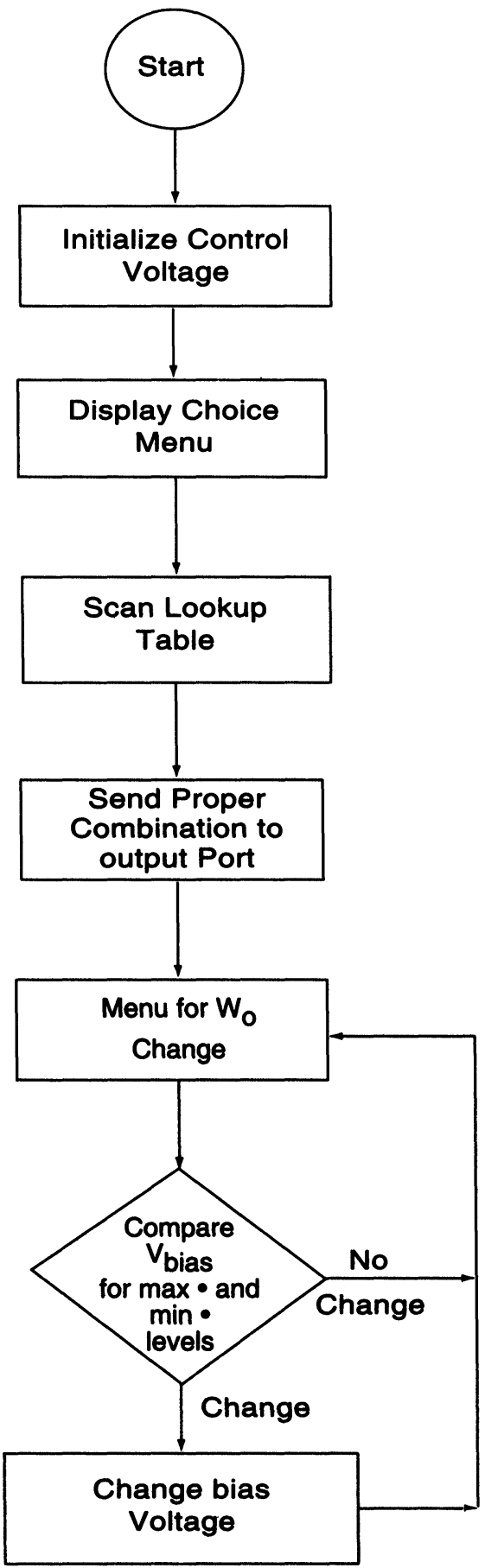

FIGURE 3 Flowchart of the proposed software. 


\section{Cut-off (Centre) Frequency Control}

a. The program goes in to a subroutine prompting the user about a change in frequency, if desired.

b. User's choice is constantly scanned using a built-in subroutine of the microcontroller to scan the keyboard.

c. When a selection for increase or decrease in frequency is made, the microcontroller goes through a routine to change the bias voltages for the OTAs.

d. Minimum and maximum levels for the voltages are checked. This check has been introduced such that not only proper filter operation is guaranted, but device protection is also taken into account.

e. The change in bias current, thus a change in g's of OTAs, is achieved by changing the value at the proper $\mathrm{D} / \mathrm{A}$.

In all of the menu routines, the user can at any time exit to the previous menu, and subsequently to main menu, where a change in the filter realization can be achieved. Thus, the software offers the user complete control over filter operation. Since the microcontroller is not involved in the actual frequency determination, there is no limitation on the input signal frequency except that imposed by the OTAs.

\section{CONCLUSION}

In this paper, a programmable multifunction filter has been presented. The proposed filter can provide three filter functions simultaneously. The filter functions as well as the parameters of the filter can be programmed using a microcontroller. It must be mentioned here that not all the functions of the microcontroller are used. Thus, it is possible to incorporate the filter circuit on the microcontroller chip. This way the unused area of the chip can be efficiently utilized to get a filter embedded in the microcontroller chip.

The proposed programmable filter is very attractive for CMOS VLSI implementation. This is attributed mainly to the use of minimum number of resistors; only four resistors for auxiliary bias current control.

\section{REFERENCE}

1. J. Wu and C.-Y. Xie, New multifunction active filter using OTAs, International Journal of Electronics, Vol. 74, 1993, pp. 235-239. 

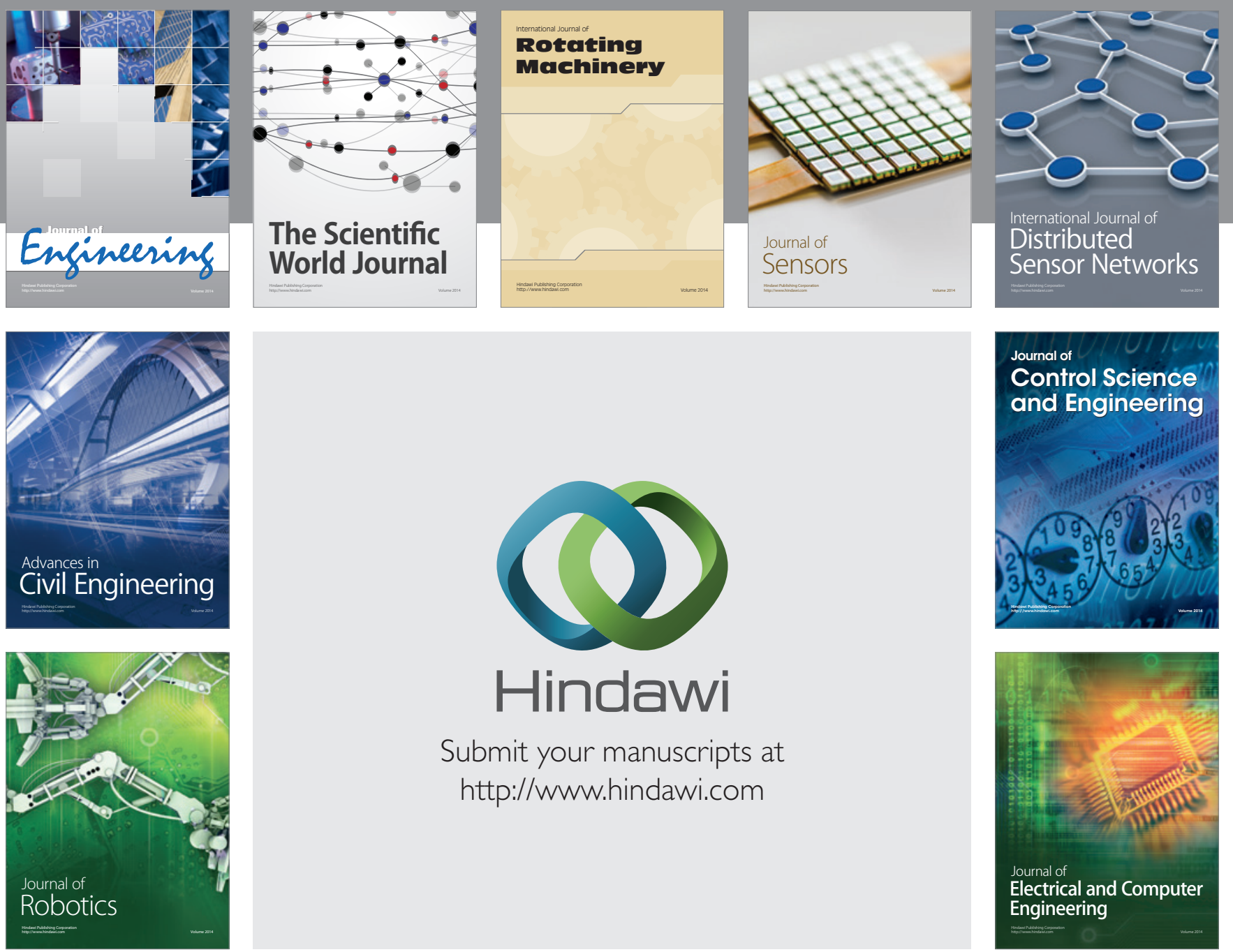

Submit your manuscripts at

http://www.hindawi.com
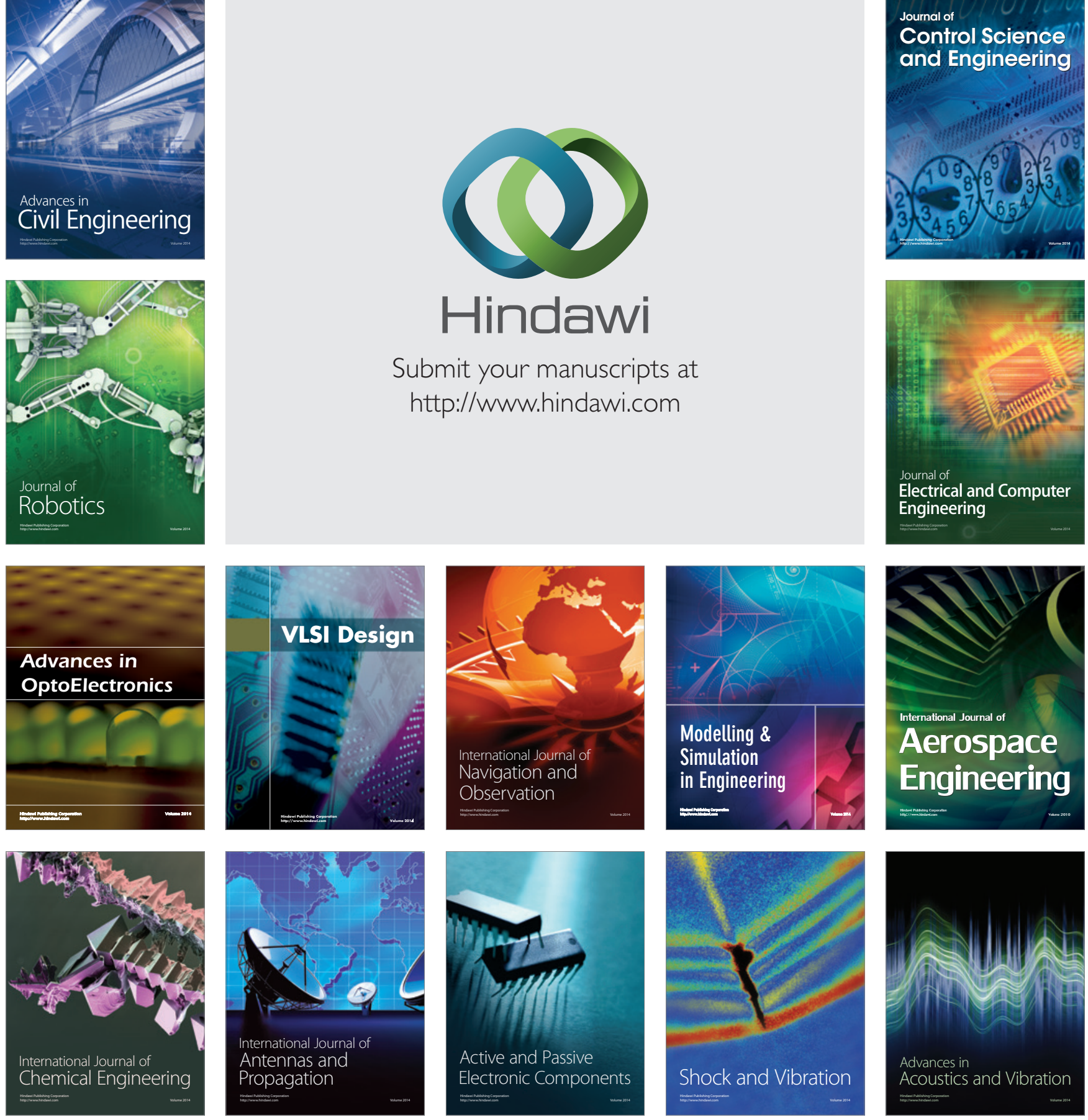